\title{
APS: UN ITINERARIO PARA LAS COMPETENCIAS EN LA ASIGNATURA DE DEPORTE ADAPTADO
}

\author{
José Manuel Puchalt Hernández \\ Universidad Católica de Valencia San Vicente Mártir (Valencia) \\ jm.puchalt@ucv.es
}

\begin{abstract}
Recepción Artículo: 1 marzo 2020
Admisión Evaluación: 4 marzo 2020

Informe Evaluador 1: 8 abril 2020

Informe Evaluador 2: 22 marzo 2020

Aprobación Publicación: 20 abril 2020
\end{abstract}

\section{RESUMEN}

En el tercer curso del Grado de Ciencias de la Actividad Física y Deporte de la Universidad Católica de Valencia se imparte la asignatura "Deporte Adaptado y Actividad Física Inclusiva", cuyo objeto de estudio son las personas con discapacidad y su relación con los diferentes ámbitos del deporte, terapéutico, educativo, competitivo y de ocio y recreación.

Para conseguir las competencias específicas de la asignatura los alumnos pueden elegir tres itinerarios distintos. Por un lado, se ofrece trabajo en grupo con exposición teórica y aplicación práctica; por otro, portafolios como trabajo individual y en tercer lugar itinerario de Aprendizaje y Servicio, en el que los alumnos por grupos buscan una institución y desarrollan en ella un programa de Aprendizaje-Servicio mediante el cual adquieren las competencias específicas de la asignatura. Este último itinerario es el que mejor cumple los objetivos de la asignatura, puesto que los alumnos se familiarizan con las personas usuarias de las instituciones donde lo aplican y se sensibilizan hacia la discapacidad, obteniendo un aprendizaje auténtico y situado, puesto que vivencian la discapacidad en primera persona. También es una metodología que denominamos "trascendente" puesto que trasciende la Universidad y repercute directamente en el entorno social más cercano, reduciendo esa brecha que a veces existe entre la Universidad y la sociedad real.

Una vez elegida la institución y solucionados los trámites administrativos, el itinerario APS consta de cuatro fases que realizan los alumnos en grupo: preparación, intervención, evaluación y difusión. Del desarrollo de todas ellas da cuenta la presente comunicación.

Palabras clave: deporte adaptado; competencias; APS; impacto social universitario

\section{ABSTRACT}

Aps: an itinerary for competences in the subject of adapted sport. In the third year of Physical Activity and Sport Sciences degree at the Catholic University of Valencia, the subject «Adapted Sport and 


\section{APS: UN ITINERARIO PARA LAS COMPETENCIAS EN LA ASIGNATURA DE DEPORTE ADAPTADO}

Inclusive Physical Activity» is taught. Its main purpose is to better know people with disabilities and their relationship with the different areas of the sport, therapeutic, educational, competitive and leisure and recreation.

In order to achieve the specific competences of the subject, students can choose three different itineraries. On the one hand, group work with theoretical exposition and practical application is offered; on other, portfolios as individual work and thirdly, the Learning and

Service itinerary, in which students by groups search for an institution and develop a ServiceLearning program in which they acquire the specific skills of the subject. This last itinerary is the one that best meets the objectives of the subject, since students become familiar with the users of the institutions where they apply it and become sensitized towards disability, obtaining authentic and situated learning, given that they experience disability in first person. It is also a methodology that we call "transcendent" since it transcends the University and directly affects the closest social environment, reducing the gap that sometimes exists between the University and real society.

Once the institution has been chosen and the administrative procedures have been solved, the S-L itinerary has three phases carried out by the students in groups: preparation, intervention, evaluation and diffusion. The development of all of them is reported in this communication.

Keywords: adapted sport; competences; APS; university social impact

\section{INTRODUCCIÓN}

Dentro del Grado de Ciencias de la Actividad Física y Deportes, en $3^{0}$ curso, como parte de la materia "Fundamentos de los Deportes", se encuentra la asignatura "Deporte Adaptado y Actividad Física Inclusiva", cuyo objeto de estudio son las personas con discapacidad y su relación con los diferentes ámbitos del deporte, terapéutico, educativo, competitivo y de ocio y recreación. Partiendo de los cambios metodológicos que se han dado en los últimos 10 años en la enseñanza superior a raíz de los acuerdos de Bolonia (2010), las metodologías activas (Fernández March, 2006) son el instrumento adecuado para el aprendizaje por competencias, en las que se le exige al alumnado un aprendizaje activo, un "aprender haciendo" (Dewey, 1995), en lugar de las metodologías tradicionales, en las que se limitaban a ser meros receptores pasivos de la información que poseía el profesor; además de los documentos normativos, las Técnicas de la Información y Comunicación (TICs) y el acceso inmediato a la información (internet), también han contribuido al cambio metodológico. Así pues, enseñar por competencias supone un aprendizaje globalizador e integrador puesto que sitúa al alumnado frente a una situación real en un contexto concreto donde aplicando sus conocimientos, capacidades, habilidades, actitudes y valores, deben resolver de manera eficaz dicha situación (Zabala y Arnau, 2007). Si además este poner a prueba al alumnado se hace en un contexto social próximo, que suponga un servicio a la comunidad, estamos ya situados en el Aprendizaje-Servicio como metodología docente. Opinamos como Batlle (2011) que el APS es una potente metodología para nuestro alumnado, puesto que la realización de un servicio a la comunidad, es una gran oportunidad de formarse en valores, poniendo de manifiesto el efecto educativo de hacer cosas para los demás, siendo una manera de superar el egocentrismo y crecer como personas.

Nuestro objeto de estudio son las personas con discapacidad, un colectivo de los más vulnerables en la sociedad actual pese a todos los cambios de perspectiva que nos está trayendo el nuevo paradigma de la inclusión, es por ello, que desde nuestra asignatura es fácil y además conveniente aplicar esta metodología APS, puesto que en todos los entornos sociales cercanos a nuestro alumnado, existen colectivos, asociaciones, instituciones que se dedican a la atención a este tipo de población, respetando siempre uno de los principios intrínsecos al APS, la voluntariedad, puesto que desde nuestra asignatura presentamos 3 diferentes itinerarios para alcanzar las competencias de la misma.

Nuestro planteamiento didáctico es el siguiente: Siendo que desarrollar y evaluar Competencias requiere prácticas, éstas pueden ser simuladas (como hacemos en muchas de las sesiones de sensibilización y de los distintos deportes adaptados, en las que adoptamos roles de personas con discapacidad) o reales. En cuanto a las reales, las cuales creemos más convenientes, desde la asignatura procuramos en la medida de lo posible dar a 
conocer deportistas con discapacidad u otras personas destacadas en cualquier faceta social que tengan entre sus características algún tipo de discapacidad, o que estén en relación con la atención a personas con discapacidad, ello lo hacemos a través de las conferencias de aula, también invitamos a nuestro alumnado a conocer diferentes programas que tiene la UCV como el Servicio de Actividad Física y Deportiva con programas específicos de personas con discapacidad o diferentes programas del Campus Capacitas. Pero partiendo del principio de voluntariedad si esas prácticas reales, se realizan fuera de la universidad, en cualquier colectivo de personas con discapacidad, éstas cobran una dimensión de servicio social, dándose los dos componentes del APS, aprendizaje por un lado y servicio social por otro, de forma que el APS permite desarrollar en nuestro alumnado las competencias de la asignatura, en contextos reales a la vez que hace un bien social. Como dice Puig (2009) el APS combina en una sola actividad aprendizajes de contenidos, desarrollo de competencias y adquisición de valores con tareas de servicio a la comunidad. Y es por ello, que ofrecemos a nuestro alumnado esta posibilidad de cursar la asignatura, escogiendo el itinerario de APS, frente a los otros dos itinerarios de Trabajo Cooperativo en Grupo con Exposición y Aplicación Didáctica Práctica o el itinerario de Portafolio.

Una última característica que queremos destacar del APS es que con esta metodología se supera la clásica dicotomía entre universidad-sociedad, entre teoría y práctica, entre conocimientos conceptuales- conocimientos prácticos, puesto que, al situar al alumnado ante problemas reales y situados en un contexto social determinado, el conocimiento conceptual, académico o teórico, se vuelve significativo y se lleva a la realidad, puesto que se aplica y se comprueba, a la vez que ayuda a mejorar la práctica. Lo que se aprende en las aulas se puede transferir a la realidad en forma de acción y permite un servicio de calidad a la comunidad. En palabras de Tapia (2006) "aprender, sirve" y "servir enseña".

Es por ello que nosotros hemos denominado al APS o también al Aprendizaje Basado en Proyectos (ABP), metodologías trascendentes, puesto que su aplicación, trasciende más allá de las aulas y repercute directamente en la realidad cercana del alumnado, frente a otras metodologías activas como el Aprendizaje Cooperativo que su acción y sus beneficios no van más allá de los alumnos que la practican.

\section{OBJETIVOS}

El objetivo de esta comunicación es dar a conocer como la metodología APS forma parte de los itinerarios para conseguir las competencias específicas de la asignatura de Deporte Adaptado y Actividades Físicas Inclusivas de $3^{0}$ de Ciencias de la Actividad Física y Deportes de la Universidad Católica de Valencia San Vicente Mártir, analizando de forma cualitativa, a través de las valoraciones que hacen los alumnos en la fase de evaluación de ésta metodología, el grado de adquisición de dichas competencias.

Para ello, presentamos a continuación los objetivos generales de la asignatura que los alumnos deben alcanzar de forma competencial, es decir, sometiéndoles a diferentes situaciones-problema que deben resolver e invitándoles a que reflexionen y valoren la relación entre discapacidad y actividad física y deportiva.

Los objetivos generales de la asignatura son:

- Introducirse en la realidad de la discapacidad, mediante la experimentación de las limitaciones y otros tipos de posibilidades metodológicas en actividad física.

- Conocer los conceptos básicos de la atención a la diversidad, especialmente del paradigma inclusivo.

- Conocer la organización y estructuras de la Actividad Física Adaptada en distintos ámbitos tanto a nivel internacional como nacional.

- Conocer y practicar las distintas modalidades del deporte adaptado.

- Manejar las estrategias inclusivas y las adaptaciones formales y planificar actividades en relación a ellas.

- Mostrar la sensibilidad que las personas con discapacidad merecen.

- Cuestionar y reflexionar sobre el modelo actual de atención a la diversidad. 


\section{APS: UN ITINERARIO PARA LAS COMPETENCIAS EN LA ASIGNATURA DE DEPORTE ADAPTADO}

\section{METODOLOGÍA}

\section{Contexto y participantes}

Al inicio del curso de la asignatura de Deporte Adaptado y Actividades Físicas inclusivas, presentamos los tres itinerarios con los que los alumnos pueden alcanzar las competencias requeridas en la misma (Figura 1), a saber, Trabajo Cooperativo en Grupo con Exposición y Aplicación Práctica; Portafolio o Intervención APS. Respetando el principio de voluntariedad, presentamos esta última como un "Reto", como un "Desafío" hacia los estudiantes que quieren implicarse más intensamente en la asignatura y en el conocimiento de las personas con discapacidad, iniciando un clima motivacional adecuado para el aprendizaje (Gamo, 2012).

\section{Instrumentos}

La Guía Didáctica de la asignatura es el instrumento que orienta y conduce al alumnado durante toda la docencia de la misma.

Como instrumentos significativos para esta comunicación extraemos de la misma, por un lado, la ficha de participación en la asignatura (figura 1), en la que el alumnado después de haber leído la Guía y habiéndose tratado en clase el modo de evaluación de cada itinerario y resuelto las preguntas y dudas que se les plantean; eligen voluntariamente uno de los 3 itinerarios. Este primer documento es obligatorio para todo el alumnado que cursa la asignatura.

Figura 1. Ficha de participación de la asignatura.

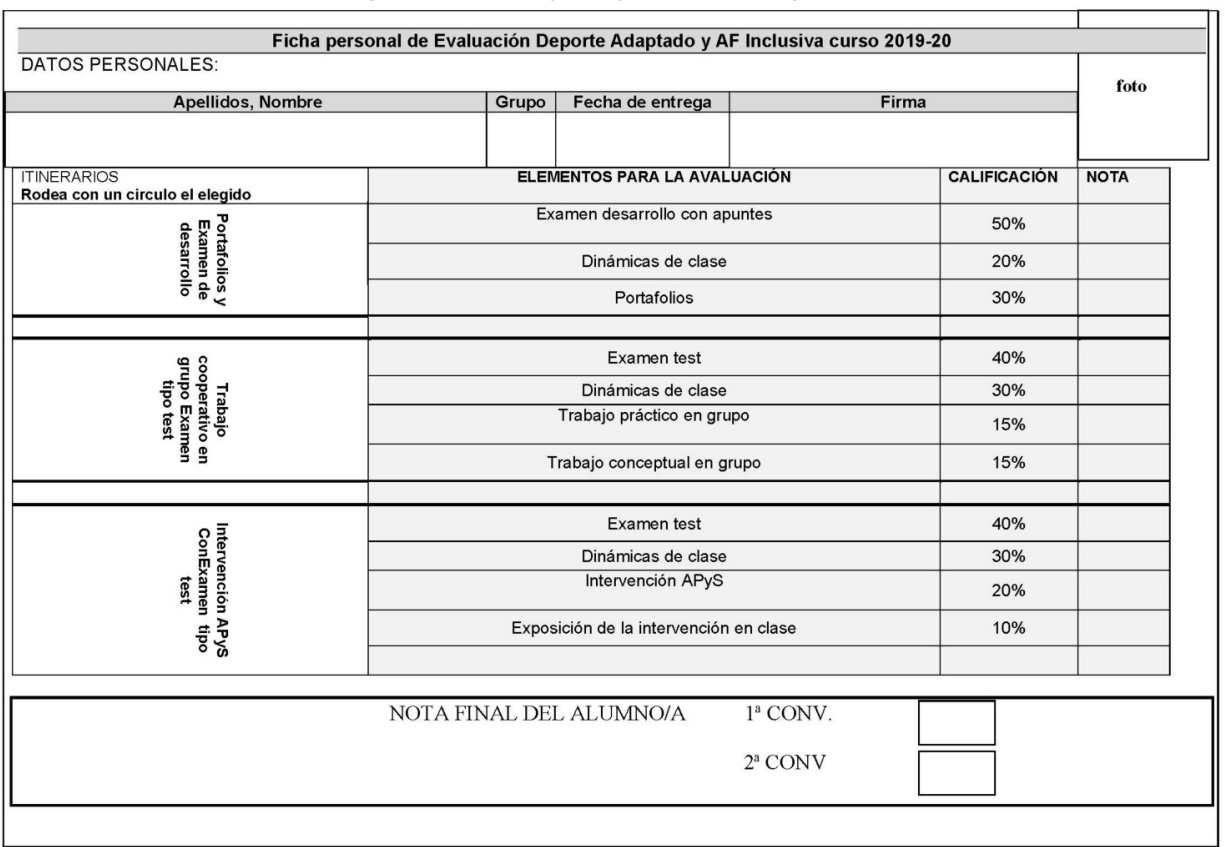

Una vez elegido el itinerario, el siguiente instrumento es de uso solo para aquellos que han elegido el itinerario APS. Se trata de la Guía para la intervención APS que se encuentra en la Guía Didáctica de la asignatura.

A continuación, mostramos dicho documento (figura 2) que está inspirado en las guías que tiene publicadas la Fundación Zerbikas (2010) 
Figura 2. Fases del APS como itinerario de la asignatura de Deporte Adaptado y Actividad Física Inclusiva

I. PREPARACIÓN:

a. Interno:

i. Constitución del grupo de trabajo para APS

ii. DAFO sobre el grupo y posterior CAME

iii. Analizar las preferencias sobre campos de acción social.

iv. Relacionados con el ámbito de las personas con discapacidad.

b. Externo:

i. Analizar e identificar entidades o instituciones donde se pueda aplicar el ApS en un entorno cercano.

ii. Establecer posibles aprendizajes que el grupo pueda llevar a cabo en dichas instituciones.

c. Contactar con las entidades o instituciones:

i. Entrevista con los responsables de las mismas.

ii. Identificar sus demandas y necesidades

iii. Proponer servicios que pueda realizar el grupo en dicha institución

iv. Consensuar y definir dentro del grupo la institución elegida y el servicio a realizar.

d. Formalizar la relación UCV con la entidad elegida a través de un documento formal.

e. Planificar la intervención.

\section{INTERVENCIÓN:}

a. Observación: Primer contacto observación de las dinámicas que habitualmente se realizan en el centro o institución (1 -2 sesiones).

b. Acercamiento-Colaboración: Los miembros del grupo empiezan a interactuar con los usuarios del centro o institución de la mano de los profesionales o responsables del mismo. Presentación a los usuarios del proyecto del grupo (1-2 sesiones mínimo)

c. Intervención directa: Puesta en práctica del proyecto con total protagonismo de los miembros del grupo. Se recomienda un diario de prácticas de todo lo acontecido y registros audiovisuales si son posibles. Reflexionar tras cada sesión como plantear o que modificaciones puede haber para la siguiente ( 4 sesiones mínimo)

III. EVALUACIÓN:

a. Interna:

i. Evaluación del trabajo en su conjunto y a cada uno de los miembros del grupo.

ii. Autoevaluarse cada miembro del grupo

iii. Reflexionar y evaluar los aprendizajes y las competencias conseguidas.

b. Externa:

i. Evaluar si el proyecto atiende a la metodología APS.

ii. Reflexionar y evaluar los resultados del servicio realizado.

iii. Solicitar reflexiones y evaluaciones a los propios usuarios del servicio y responsables de la institución.

iv. Proyectar posibles perspectivas de futuro del servicio. 


\section{DIFUSIÓN:}

a. El grupo realizará una memoria donde aparezcan todos los puntos anteriores tras la realización del proyecto APS y la entregará al profesor. a la vez que elaborará una presentación audiovisual para exponerla a los compañeros de clase.

b. Es posible también la difusión del proyecto al resto de miembros de la comunidad de la UCV a través de las Jornadas internas de APS.

\section{DESARROLLO DEL ITINERARIO APS. FASES}

Tal y como recoge la Guía del Itinerario APS, este consta de cuatro fases: Preparación, Intervención, Evaluación y Difusión.

A continuación, vamos a detallar aquello que se realiza en cada una de dichas fases.

\section{Fase de Preparación}

En esta primera fase, Ios alumnos que han elegido el itinerario APS, se organizan en grupos de trabajo de 3 a 5 personas, constituyendo formalmente a través de documentos internos el grupo de trabajo. A continuación, viene la elección del centro o institución donde se debe llevar a cabo el mismo. Dicha elección corre a cargo del grupo, y es una de las primeras tareas que deben de afrontar. Esta búsqueda ya supone un primer paso de acercamiento a la realidad social del mundo de la discapacidad, aprendiendo como resuelve institucionalmente nuestra sociedad el problema de las personas con discapacidad. Una vez consensuado con un centro o institución una intervención APS, formalizados a través de documentos facilitados por la UCV dicha relación, con el consentimiento por parte de la dirección del centro receptor, del acceso e intervención de nuestros alumnos en su centro 0 institución. La UCV acredita a través de documentos la identidad del alumnado como propio de la UCV y la intención de la intervención como parte de un trabajo de nuestra asignatura.

\section{Fase de Intervención}

La fase de Intervención se subdivide a su vez en tres subfases:

- Observación

- Colaboración

- Intervención, propiamente dicha.

Aunque la parte de planificación de la intervención en la Guía, forma parte de la fase de Preparación, la verdad es que esta se concreta en esta segunda fase, cuando se tiene un conocimiento real de la institución y de los usuarios de la misma.

La subfase de Observación consta de 1-2 sesiones de trabajo, en las que el alumnado observa que tipo de trabajo se hace con los usuarios de la misma, sirviendo para ajustar las posibilidades de la intervención a las características de los usuarios.

La subfase de Colaboración consta también de 1-2 sesiones de trabajo, donde nuestros alumnos colaboran con el personal técnico de la institución en el desarrollo de las tareas que habitualmente se realizan allí.

Por último, la subfase de Intervención directa, debe constar de 4 sesiones de trabajo mínimo, siendo la subfase más importante de todas, pues es la que le da el sentido al APS, en ella nuestro alumnado tras haber observado y colaborado, propone tanto a los técnicos y dirección del centro como al profesor de la asignatura el tipo de intervención a realizar, tras haber evaluado que pueden ellos aportar como expertos en actividad física y deporte a esa institución. Tras autorizarse la intervención por parte, tanto de la institución como del profesor de la UCV, se lleva a cabo esta fase esencial del proceso. 


\section{Fase de Evaluación}

Esta tercera fase es fundamental para que exista aprendizaje, 0 al menos para hacer conscientes al alumnado del mismo. En este sentido, esta fase la dividimos en dos apartados, uno la evaluación interna, en la que se atiende más a los aspectos de aprendizaje del APS y donde se reflexiona y se valora Ios aprendizajes adquiridos, a la vez que sirve de criterio de calificación para la asignatura por parte del profesor. Y por otro lado, la evaluación externa, que atiende más a los aspectos del servicio dado a la institución o colectivo donde se ha llevado a cabo el APS.

En la evaluación interna hacemos reflexionar a los alumnos sobre su implicación en el proyecto, sobre las competencias que han adquirido y sobre los sentimientos y valores que han aflorado en ellos durante este proceso de interacción con las personas con discapacidad. Para ello, utilizamos la autoevaluación y la coevaluación del grupo utilizando como instrumentos, tanto el registro anecdótico o diario de prácticas que ha ido elaborando el grupo como plantillas o escalas de valoración que les proporcionamos para dicha reflexión, como documento final de dicha reflexión está la memoria de la intervención APS que deben presentar al profesor para ser evaluada y calificada (heteroevaluación). Junto a esta memoria, también será calificada por parte del profesor, la exposición y el documento de soporte para la misma que el grupo utilizada para dar a conocer al resto de la clase su trabajo de intervención APS (fase de difusión). Con todo, la Intervención APS tiene un valor del 30\% del total de la nota de la asignatura repartida del siguiente modo: 0'50\% tutorías de seguimiento del trabajo (2 obligatorias); Autoevaluación del grupo 0’50\%; Presentación de la memoria de la intervención 10\%; exposición y difusión al resto de la clase 10\%. Entre los criterios de calificación que utiliza el profesor para valorar todo el proceso de intervención APS están, el nivel de implicación del mismo, respetando las 6 sesiones mínimo de las subfases de intervención; el que haya habido una verdadera aportación al centro, es decir que se haya realizado un servicio aprovechando las fortalezas de ser expertos en actividad física y deporte, en lugar de que se haya simplemente colaborado con la institución a realizar las tareas habituales que tiene programadas la institución. En este caso el rol desempeñado por nuestro alumnado se acercaría más a lo que es "voluntariado" que lo que es "servicio". Por último, valoramos aquellos aprendizajes adquiridos viendo el grado de sensibilización y empatía que se ha creado hacia las personas con discapacidad y los conocimientos reales sobre cómo actuar y que actividades pueden desarrollar las personas con las que han trabajado.

Por otro lado, en la evaluación externa, pedimos que tanto a los usuarios (si es posible) como a los responsables de la institución que nos den un "feedback" de lo que ha supuesto la intervención de nuestro alumnado en su centro, siendo como hemos dicho anteriormente la parte de servicio lo que se evalúa de esta forma. Normalmente no se utilizan documentos formales sino entrevistas o conversaciones informales con el profesor a la vez que éste agradece la deferencia de haber dejado a los alumnos de la UCV intervenir en su institución.

\section{Fase de Difusión}

El itinerario APS finaliza con la entrega de la memoria de la actividad realizada y la exposición al resto de compañeros de clase de la experiencia vivida, utilizando medios audiovisuales como vídeos o presentaciones multimedia. En las mismas apreciamos en la mayoría de los casos la gran satisfacción e implicación en el trabajo realizado y cuál ha sido el proceso de sensibilización hacia el universo de la discapacidad.

Algunos de estos trabajos han sido difundidos más allá del aula clase, como en las jornadas de APS que organiza la propia UCV, o en congresos como el I Congreso Internacional: Cátedra abierta Scholas Occurrentes, organizado también por la UCV en Valencia en 2017.

\section{CONCLUSIONES}

Al finalizar la intervención APS, todos aquellos alumnos que eligieron este itinerario, que afrontaron el Reto que les planteamos al principio de la asignatura, cuentan en la memoria final, que el proceso que experimentaron paso del temor y la inseguridad que sentían al principio al enfrentarse a una experiencia desconocida, a unas personas diferentes con las que nunca habían interactuado, a la satisfacción de verse capaces de trabajar y generar 


\section{APS: UN ITINERARIO PARA LAS COMPETENCIAS EN LA ASIGNATURA DE DEPORTE ADAPTADO}

actividades para y con ellos. A través de la reflexión sobre la acción, lo que Martín (2009) denomina "Pedagogía de la reflexión", hacemos conscientes a nuestro alumnado de todo lo que han aprendido y como ha cambiado su percepción hacia el mundo de la discapacidad; El hecho de sentirse útiles, competentes, solidarios por haber superado el reto que suponía enfrentarse al APS, hace que les suba su autoestima, al ver los resultados de su acción inmediata y cabe decir también, que estas experiencias han despertado más de una vocación entre nuestro alumnado viendo un futuro campo profesional en la relación entre la actividad física y el deporte con el ámbito de la discapacidad.

A la pregunta de si con el itinerario APS se adquieren las competencias específicas de la asignatura de Deporte Adatado y Actividad Física Inclusiva, que están sintetizadas en los objetivos generales de la misma, la respuesta es un contundente sí, por las siguientes razones:

- Puesto que el nivel de sensibilización hacia la discapacidad que muestran los alumnos del itinerario de APS, es muy elevado y así lo demuestran en sus exposiciones de lo que ha sido su APS a los demás compañeros de clase donde llegan a emocionarse y a emocionarlos a su vez. Frases como "Vinimos a enseñarles cosas sobre deporte y son ellos los que nos han enseñado cosas sobre la vida", recogidas en alguna memoria de APS dan fe de ello.

- Porque demuestran en sus exposiciones que las estrategias y adaptaciones que han aplicado en su intervención les han servido para conocer mejor y saber actuar en diferentes actividades físicas y deportivas con personas con discapacidad, siendo más competentes para ello.

- También porque la búsqueda de un centro donde realizar la intervención APS les ha dado una visión de las instituciones, centros, asociaciones y demás organizaciones que giran alrededor del ámbito de la discapacidad en su entorno social más cercano.

- Y por último porque la memoria que presentan tras la intervención, realizando una reflexión sobre la acción (Martin, 2009) les hace, por un lado, aflorar todo lo que han aprendido y por otro, ser críticos con el actual sistema atencional hacia la discapacidad, aportando y viendo en que aspectos los profesionales de la Actividad Física y Deporte pueden contribuir a mejorar la atención a las personas con discapacidad.

Coincidimos plenamente con la opinión del doctor Puig (2009) en que la metodología APS tiene un alto valor formativo por la forma integral que tiene de adquisición de las competencias requeridas en nuestra asignatura. Además, para nosotros, el valor añadido que tiene es lo que denominamos su carácter "trascendente", puesto que su aplicación trasciende de lo que sucede en el aula de la Universidad al seno mismo de la comunidad más cercana.

\section{REFERENCIAS BIBLIOGRÁFICAS}

Batlle, R. (2011). ¿De qué hablamos cuando hablamos de aprendizaje-servicio. Crítica, 972, 49-54.

Dewey, J. (1995). Democracia y educación: una introducción a la filosofía de la educación. Ediciones Morata.

Fernandez March, A. (2006). Metodologías activas para la formación de competencias. Educatio siglo XX/(24), $35-56$.

Fundación Zerbikas (2010). Guía práctica de aprendizaje servicio para jóvenes. Disponible en: http://www.aprendizajeservicio.net/guias/castellano/index.html

Gamo, J. R. (2012). La neuropsicología aplicada a las ciencias de la educación: Una propuesta que tiene como objetivo acercar al diálogo pedagogía/didáctica, el conocimiento de las neurociencias y la incorporación de las tecnologías como herramientas didácticas válidas en el proceso de enseñanza-aprendizaje. J. Navarro, M. ํㅜ. Fernández, FJ Soto, y F. Tortosa (coords.), Respuestas flexibles en contextos educativos diversos. Murcia: Consejería de Educación, Formación y Empleo.

Martín, X.; Rubio, L. (Coord.) (2006). Experiencies d'aprenentatge servei. Barcelona: Octaedro.

Ministros Europeos de Educación. (1999). Declaración de Bolonia.Declaración conjunta de los Ministros Europeos de Educación. Recuperado el 16 de mayo de 2014, de http://www.eees.es/pdf/Bolonia_ES.pdf 
Puchalt, J.M. (2019). Guía Didáctica de Deporte Adaptado y Actividad Física con Necesidades Educativas Específicas. Grado en CC de la Actividad Física y Deportes. UCV San Vicente Mártir de Valencia.

Puig, J.Mạ. (Coord.) (2009). Aprendizaje Servicio (ApS). Educación y Compromiso Cívico. Barcelona: Graó.

Tapia, M.N. (2006). Aprendizaje y servicio solidario. En el sistema educativo y las organizaciones juveniles. Buenos Aires: Ciudad Nueva.

Zabala, A.; Arnau, L. (2007) ¿Cómo aprender y ensañar competencias?. Barcelona: Graó. 
